\title{
Risk Analysis Based on Investment Structure Model
}

\author{
Jing Wang \\ School of Economics and Management, Nanjing University of Science and Technology, Nanjing \\ 210094, China \\ wjmaps@163.com
}

Keywords: Portfolio Optimization, Pair Copula, SV-t model, Risk , CVaR

\begin{abstract}
In order to solve the problem of investment structure optimization under non-normal and nonlinear correlation, a new investment structure optimization method based on Pair Copula-SV-t is proposed. According to the existing optimization model of $\mathrm{t}$-Copula-SV-t, the Pair Copula decomposition model is introduced into it, and the Pair Copula-SV-t model is constructed with the SV-t model to fit the expected return rate $Y^{\boldsymbol{k}}$ of the asset. The feasibility and effectiveness of the model are verified by corresponding empirical analysis.
\end{abstract}

\section{Introduction}

In the financial market, risk is the first factor affecting the results of the investment structure model. Therefore, how to accurately quantify the risk is the core part. In the modern portfolio theory framework, it is feasible to use modern linear correlation risk metrics when the asset return sequence is required to follow a normal distribution. However, financial time series usually exhibit non-normality and nonlinear correlation, so traditional linear correlation-based risk measurement methods cannot correctly measure investment structure risks. Therefore, to solve the problem of investment structure optimization under non-normal and nonlinear correlation, the optimal weight and corresponding risk value are obtained. Firstly, a joint distribution function based on the Pair Copula-SV-t model to simulate the return on investment structure is constructed. The Pair Copula decomposition model is used to describe the correlation structure between each asset. The model is Monte Carlo simulation, and the n-dimensional random variable is obtained. Then the edge distribution function of the investment structure yield is fitted according to the SV-t model. The variable is transformed to obtain the yield series $Y^{k}$, and finally the mean-CVaR investment structure optimization model is used for risk measurement. 


\section{Methodology}

\subsection{Pair Copula decomposition model}

Pair Copula decomposition is the product of decomposing a high-dimensional density function into a binary Copula function and an edge probability density function.Because the vine-based pair Copula decomposition model has great flexibility and superiority in constructing multivariate Copula functions, it is superior to traditional methods in portraying asset-related structures. The recursive formula of the conditional density function is:

$$
f(x \mid v)=c_{x v j \mid v_{-j}}\left\{u_{x \mid v_{-j}}, u_{v_{j} \mid v_{-j}}\right\} f\left(x \mid v_{-j}\right\}
$$

where $v=\left[v_{1}, \ldots, v_{j}, v_{j+1}, \ldots, v_{d}\right], v_{-j}=\left[v_{1}, \ldots, v_{j-1}, v_{j+1}, \ldots, v_{d}\right]$

Because the Pair Copula decomposition has many different expressions, Kurowicka and Cooke introduced the concept of "regular vines" in order to effectively organize each decomposition. Commonly available $C$ vines and D vines. But which one is more suitable for characterizing structure between variables? We use the AIC and BIC guidelines to determine.The n-element joint density function of $\mathrm{D}$ vine is

$$
f\left(x_{n}\right)=\prod_{k=1}^{n} f\left(x_{k}\right) \prod_{j=1}^{n-1} \prod_{i=1}^{n-1} c_{i, i+j \mid i+1, \ldots, i+j-1}\left\{u_{i \mid i+1, \ldots, i+j-1} u_{i+j \mid i+1, \ldots, i+j-1}\right\}
$$

The $\mathrm{n}$ - element joint density function of $\mathrm{C}$ vine

$$
f\left(x_{n}\right)=\prod_{k=1}^{n} f\left(x_{k}\right) \prod_{j=1}^{n-1} \prod_{i=1}^{n-1} c_{j, i+j \mid i+1, \ldots, j-1}\left\{u_{j \mid 1, \ldots, j-1,} u_{i+j \mid 1, \ldots, j-1}\right\}
$$

\subsection{SV-t model}

Compared with the SV model, the SV-t model can better describe the edge distribution of the return on assets. it is clear that the SV-t model is more reasonable to sovle the "spike and thick tail" phenomenon that is common in financial market returns.

\subsection{Optimization model of investment structure based on mean-CVaR}

$\mathrm{CVaR}$ reflects the excess average loss, which means that the information before t time has been known, and the mean value of the loss exceeds VaR under the confidence level of $\beta$.The investment structure optimization model based on mean-CVaR :

$$
\min \alpha+\frac{1}{q(1-\beta)} \sum_{k=1}^{q} u^{k}
$$

s.t. $X^{\prime} Y^{k}+\alpha+u^{k} \geq 0, u^{k} \geq 0, \quad x_{i} \geq 0, \frac{1}{q} X^{\prime} \sum_{k=1}^{q} Y^{k} \geq \rho, \sum_{i=1}^{n} x_{i}=1$

\subsection{Optimization model of investment structure based on Pair Copula-sv-t model}

Steps of the investment structure optimization model based on $\mathrm{D}$ vine:

1) Randomly generate $\mathrm{n}$ variables $\left(x_{1}, \ldots, x_{n}\right), x_{i} \sim U(0,1), i=1, \ldots, n, x_{1}=v_{1}$;

2)According to equations (1)and (2), random variables $\left(v_{2}, \ldots, v_{n}\right)$ obeying the Pair Copula 
decomposition model can be generated;

3) Repeat step 2) q times to get q simulation results of random vectors $\left(v_{2}, \ldots, v_{n}\right)$

4) According to the edge distribution function $F_{i}\left(r_{i}\right)$ obtained by fitting the SV-t model, the random variable $\left(v_{2}, \ldots, v_{n}\right)$ is transformed, and the yield vector $\left(r_{1}, \ldots, r_{n}\right)$ of the $n$ kinds of assets, that is, the investment structure, can be obtained, where $r_{i}=F_{i}^{-1}\left(v_{i}\right), i=1, \ldots, n$

5) From the previous step, the expected yield series $Y^{k}$ can be obtained, where $Y^{k}$ is the kth simulation result $(k=1, \ldots, q)$ of the yield vector $\left(r_{1}, \ldots, r_{n}\right)$ of $\mathrm{n}$ kinds of assets, and then $Y^{k}$ substituting equation (4) can calculate the $\mathrm{VaR}, \mathrm{CVaR}$ value and optimal weight of the investment structure.

The steps of $\mathrm{C}$ vine are similar to that of $\mathrm{D}$ vine, except that the simulated yield sample data are different. In other words, the model applicable to $\mathrm{C}$ vine adopts equation (3) to solve $\mathrm{v}$ in the iterative formula $\left(v_{2}, \ldots, v_{n}\right)$

\section{Empirical analysis}

To verify the feasibility and effectiveness of the constructed Pair copula-sv-t model, this paper takes the investment structure of the three indexes of CSI 300, SSE 50 and CSI 500 as examples to measure the risk. The sample interval selected is the daily closing price $P_{t}$ of the three indices from January 4, 2015 to December 31, 2017, totaling 1455.

\subsection{Parameter Estimation and Test of SV-t Model}

First, the parameters of SV-t model are estimated. We sampled 5000 times in a cycle, then got rid of the first 1000 unconverged iterations, and selected the remaining 4000 times as the sampling results, and performed K-S test. The results are shown in Table 1.

Table 1 SV-t estimation results and K-S test

\begin{tabular}{|c|c|c|c|c|c|c|}
\hline & $\mu$ & $\varphi$ & $\sigma_{\eta}$ & $v$ & $\begin{array}{l}\mathrm{P} \text { value of the } \\
\text { residual term }\end{array}$ & $\begin{array}{l}\text { P value of random } \\
\text { disturbance term }\end{array}$ \\
\hline CSI 300 & $\begin{array}{l}0.8869 \\
(0.03)\end{array}$ & $\begin{array}{l}0.9841 \\
(0.04)\end{array}$ & $\begin{array}{l}0.0570 \\
(0.003)\end{array}$ & $\begin{array}{c}15.1487 \\
(0.09)\end{array}$ & 0.8112 & 0.4988 \\
\hline SSE 50 & $\begin{array}{l}0.6719 \\
(0.02)\end{array}$ & $\begin{array}{l}0.9849 \\
(0.07)\end{array}$ & $\begin{array}{c}0.0950 \\
(0.001)\end{array}$ & $\begin{array}{c}15.0860 \\
(0.06)\end{array}$ & 0.7390 & 0.1434 \\
\hline CSI 500 & $\begin{array}{l}0.8042 \\
(0.02)\end{array}$ & $\begin{array}{l}0.9748 \\
(0.05)\end{array}$ & $\begin{array}{c}0.0934 \\
(0.002)\end{array}$ & $\begin{array}{c}17.4838 \\
(0.03)\end{array}$ & 0.6450 & 0.9227 \\
\hline
\end{tabular}

From the K-S test value in Table 1, it can be known that all parameters of the Pair Copula function are estimated to be greater than 0 , which indicates that the three index yield series have strong correlations at the upper and lower tails, and their values are close to zero. This shows that the three index's yield series tend to be independent when they are outside the tail. It is indicated that the SV-t model can better fit the logarithmic rate of return time series of each index.

\subsection{Parameter estimation and validation of the Pair Copula model}

First, the parameter estimation of the Pair Copula model based on the two rattan structures was calculated. The results are shown in Table 2. HS, SZ, and ZZ are used to represent the CSI 300 Index, the SSE 50 Index and the CSI 500 Index respectively, and $\rho$ and $v$ are correlation coefficients and 
degrees of freedom, respectively.

Table 2 Estimation results under $C$ vine and $D$ vine

\begin{tabular}{|c|c|c|c|c|}
\hline \multirow{2}{*}{ Parameter } & \multicolumn{2}{|c|}{ C vine } & \multicolumn{2}{|c|}{ D vine } \\
\hline & Initial value & Final value & Initial value & Final value \\
\hline$\rho_{H S S Z}$ & 0.168 & 0.1695 & 0.550 & 0.5560 \\
\hline$\rho_{\text {SZZZ }}$ & 0.505 & 0.5150 & 0.168 & 0.1695 \\
\hline$\rho_{H S Z Z \mid S Z}$ & 0.373 & 0.3697 & 0.220 & 0.2200 \\
\hline$v_{H S S Z}$ & 9.28 & 9.83 & 58.09 & 51.998 \\
\hline$v_{S Z Z Z}$ & 10.46 & 10.97 & 9.28 & 9.83 \\
\hline$v_{H S Z Z \mid S Z}$ & 67.36 & 68.66 & 70.00 & 69.28 \\
\hline$M L E$ & 419.954 & 419.849 & 419.634 & 419.667 \\
\hline
\end{tabular}

Then, $A I C_{D}>A I C_{C}, B I C_{D}>B I C_{C}$ indicating that the $C$ vine fitting effect is better, so we choose the Pair Copula decomposition model based on $\mathrm{C}$ vine.

\subsection{CVaR values and optimal weights at different confidence levels}

Investment structure yield rate sequence $Y^{k}$ based on Pair Copula-SV-t model and optimization study by formula (4). In this paper, $\mathrm{VaR}$ and $\mathrm{CVaR}$ values at different confidence levels are calculated respectively, and the optimal investment structure weight is given. The results are shown in Table 3.

Table 3 CVaR and optimal weight

\begin{tabular}{cccccc}
\hline Confidence level & CSI 300 & SSE 50 & CSI 500 & VaR & CVaR \\
\hline $90 \%$ & 0.3277 & 0.6723 & 0.0000 & 0.1158 & 0.1448 \\
$95 \%$ & 0.2936 & 0.6525 & 0.0538 & 0.1885 & 0.2065 \\
$99 \%$ & 0.2608 & 0.5682 & 0.1710 & 0.2475 & 0.2884 \\
\hline
\end{tabular}

As shown in table 3,CVaR is always greater than VaR, and as the confidence level increases, VaR and CVaR also increase accordingly, which indicates that CVaR considers the loss to exceed VaR and is more sensitive to the risk.

Similarly, we can see from the optimal weight of the investment structure that the optimal portfolio is mainly invested in the CSI 300 Index and the SSE 50 Index, with a small amount of the CSI 500 Index. This reflects that the overall risk of the CSI 300 Index and the SSE 50 Index is smaller than that of the CSI 500 Index. The weighting trend of the CSI 500 Index is opposite to that of the CSI 300 and the SSE 50 Index.

\subsection{Comparison of time-varying CVaR values of the multivariate t-Copula-SV-t model and Pair Copula-SV-t model}

Finally, in order to illustrate the superiority of the Pair copula-SV-t model, the model will be compared with the multivariate t-copula-SV-t model. The results of t-copula-SV-t model are shown in Table 4,5. 
Table 4 Estimation results of t-Copula

\begin{tabular}{ccc}
\hline Parameter & Initial value & Final value \\
\hline$\rho_{\text {HSSZ }}$ & 0.55 & 0.559 \\
$\rho_{\text {SZZZ }}$ & 0.168 & 0.173 \\
$\rho_{\text {HSZZ }}$ & 0.22 & 0.274 \\
$M L E$ & 410.45 & 411.84 \\
\hline
\end{tabular}

Table 5 CVaR and optimal weight based on the t-copula model

\begin{tabular}{cccccc}
\hline Confidence level & CSI 300 & SSE 50 & CSI 500 & VaR & CVaR \\
\hline $90 \%$ & 0.4748 & 0.5064 & 0.0188 & 0.0322 & 0.0650 \\
$95 \%$ & 0.4699 & 0.5111 & 0.0190 & 0.0516 & 0.0896 \\
$99 \%$ & 0.4516 & 0.5188 & 0.0296 & 0.1121 & 0.1598 \\
\hline
\end{tabular}

Comparing Table 5 with Table 3, it can be seen that with the increase of confidence, the risk value under the Pair Copula model is greater than the risk value under the t-Copula model, indicating that the distribution and tail feature of the model can be better characterized by the Pair Copula model.

\section{Conclusions}

To solve the problem of investment structure optimization under non-normal and nonlinear correlation, a new method based on Pair Copula-SV-t investment structure model is established. The Pair Copula decomposition model is used to characterize the correlation structure between each asset, and Monte Carlo simulation is performed to obtain n-dimensional random variables.According to the edge distribution function of the investment structure return rate fitted by the SV-t model, the n-dimensional random variable is transformed to obtain the yield series $Y^{k}$.The mean-CVaR investment structure optimization model is used to measure the risk, and the optimal weight of the investment structure and the corresponding $\mathrm{VaR}$ and $\mathrm{CVaR}$ values are obtained.Finally, the feasibility and effectiveness of the risk measurement method based on Pair Copula-SV-t model are verified by empirical analysis.

\section{References}

[1] D. Ling. Investment Structure Optimization base on Pair Copula-GHRCH- EVT-CVaR. Changsha, Hunan University.2(2011) 22-23.

[2] Yanzi Li.Risk Analysis of Portfolio Investment Based on Monte Carlo Simulation. New Economy.6(2016) 38-39

[3] Xuegong Zhang.Analysis of Financial Market Correlation Based on Pair Copula-SV-t Model. Journal of Finance and Accounting. 18(2016) 76-78.

[4] Xiangdong Liu,Cheng Liu. High-dimensional combined risk measurement based on M-Copula-SV-t model. CMS. 25(2017) 3-4.

[5] Bacon,C.R. Practical Portfolio Performance Measurement and Attribution. Journal of Finance. (2015)104-105

[6] Xingwei Zhao. Bayes estimation and empirical analysis of SV model based on MCMC method. China University of Mining. 51(2017) 3-4. 